\title{
Solving Polluted Lakes System by using Perturbation-Iteration Method
}

\author{
M. Khalid \\ Department of Mathematical Sciences \\ Federal Urdu University of Arts, Sciences \& Techonology \\ University Road, Karachi-75300, Pakistan \\ Faheem Zaidi \\ Department of Mathematical Sciences \\ Federal Urdu University of Arts, Sciences \& Technology \\ University Road, Karachi-75300, Pakistan
}

\author{
Mariam Sultana \\ Department of Mathematical Sciences \\ Federal Urdu University of Arts, Sciences \& Technology \\ University Road, Karachi-75300, Pakistan \\ Fareeha Sami Khan \\ Department of Mathematical Sciences \\ Federal Urdu University of Arts, Sciences \& Technology \\ University Road, Karachi-75300, Pakistan
}

\begin{abstract}
Water pollution is a major global problem that requires ongoing evaluation and revision of water resource policies at all levels, in order to create a healthy living environment. Differential equations are an effective way to analyze such situations. In this paper a system of linear equations with interconnecting pipes is considered for analyzing the pollution of system of lakes through differential equations. Perturbation-iteration method is used to compute an approximate solution of three input models i.e. periodic, linear step model and exponentially decaying model. The fourth order RungeKutta method $(R K 4)$ numerical solution of the lakes system problem is used as a reference to compare with the analytical approximations showing the high accuracy of the results.
\end{abstract}

\section{Keywords:}

Water Pollution, Pollution of System of Lakes, Perturbation Iteration Method, Analytical Approximations

\section{INTRODUCTION}

Perturbation methods are being used effectively for over many decades in solving many algebraic equations, differential equations and integro-differential equations. They have also opened new doors to understand the structure of many applied problems. But still a very major problem with this method is the selection of parameter. This small parameter selection whether introduced artificially or it is the part of physical problem, always restricts the range of validation of the results.

To overcome this parameter selection problem, Boyaci \& Pakdemirli [1-3] developed a new perturbation-iteration method for algebraic equations. Then it was applied to differential equations and system of non linear differential equations [4] and obtained accurate convergence compared to exact solutions if available. Recently this method has been applied on many different problems and differential equations such as Bratu type equations, linear and nonlinear differential equations and systems, heat equations, Fredholm and Volterra integral equations and obtained satisfactory results [4-8] Basic idea of perturbation method is to combine Taylor series expansion and perturbation expansion and generate a new iteration series. [1, 2] introduced expansion and correction terms of only first derivatives in the Taylor Series expansion, i.e. $n=1, m=1$ and one correction term in the perturbation.

The algorithm is named $P I A(1,1)$. Consider the following system of first-order differential equations.

$$
H_{k}\left(\dot{x}_{k}, x_{j}, \epsilon, t\right)=0 ; k=1,2, . ., K ; j=1,2, . ., K
$$

where $k$ is a representative of the number of differential equations in the system. For example, $k=1$ for a single equation. In the open form, the system of equations is

$$
\begin{aligned}
H_{1}= & H_{1}\left(\dot{x}_{1}, x_{1}, x_{2}, x_{3}, \ldots, x_{K}, \epsilon, t\right)=0 \\
H_{2}= & H_{2}\left(\dot{x}_{2}, x_{1}, x_{2}, x_{3}, \ldots, x_{K}, \epsilon, t\right)=0 \\
H_{3}= & H_{3}\left(\dot{x}_{3}, x_{1}, x_{2}, x_{3}, \ldots, x_{K}, \epsilon, t\right)=0 \\
& \cdot \\
& \cdot \\
& \cdot \\
H_{k}= & H_{k}\left(\dot{x}_{k}, x_{1}, x_{2}, x_{3}, \ldots, x_{K}, \epsilon, t\right)=0
\end{aligned}
$$

Assume an approximate solution of the system

$$
x_{k, n+1}=x_{k, n}+\epsilon x_{k, n}^{c}
$$

with one correction term in the perturbation expansion. The subscript $n$ represents the $n^{\text {th }}$ iteration over this approximate solution. The system can be approximated with a Taylor series expansion in the neighborhood of $\epsilon=0$ as

$$
H_{k}=\sum_{m=0}^{M} \frac{1}{m !}\left[\left(\frac{d}{d \epsilon}\right)^{m} H_{k}\right]_{\epsilon=0} \epsilon^{m} ; k=1,2, . ., K
$$


where

$$
\frac{d}{d \epsilon}=\frac{\partial \dot{x}_{k, n+1}}{\partial \epsilon} \frac{\partial}{\partial \dot{x}_{k, n+1}}+\sum_{j=1}^{K}\left(\frac{\partial x_{j, n+1}}{\partial \epsilon} \frac{\partial}{\partial x_{j, n+1}}\right)+\frac{\partial}{\partial \epsilon}
$$

is defined for the $(n+1)^{t h}$ iterative equations

$$
F_{k}\left(\dot{x}_{k, n+1}, x_{j, n+1}, \epsilon, t\right)=0
$$

substituting Eq.(5) into Eq. (4), an iterative equation is obtained;

$$
\begin{array}{r}
H_{k}=\sum_{m=0}^{M} \frac{1}{m !}\left[\left(\dot{x}_{k, n}^{c} \frac{\partial}{\partial \dot{x}_{k, n+1}}+\sum_{j=1}^{K}\left(x_{j, n}^{c} \frac{\partial}{\partial x_{j, n+1}}\right)+\right.\right. \\
\left.\left.\frac{\partial}{\partial \epsilon}\right)^{m} H_{k}\right]_{\epsilon=0} \times \epsilon^{m}=0 \text { where } k=1,2, . ., K
\end{array}
$$

Which is a first-order differential equation and can be solved for the correction terms $x_{k, n}^{c}$. Then, using Eq. (3), the $(n+1)^{t h}$ iteration solution can be found. Iterations are terminated after a satisfactory approximation is obtained.

Note that for a more general algorithm, $n$ correction terms instead of one can be taken in expansion (3) which would then be a PIA $(n, m)$ algorithm. The algorithm can also be used generally and be applied to a differential equation system having arbitrary order of derivatives. Within the scope of this paper, only a case $n=m=1$ is considered for the sake of simplicity, because more Algebra is involved in constructing iteration solutions for $P I A(1,2)$ and $P I A(1,3)$ as compared to $P I A(1,1)$. We have computed the numerical results by the well-known symbolic software "Mathematica 9.0"

\section{MODEL OF SYSTEM OF LAKES}

Model of pollution of system of lakes is discussed in detail in [9]. Each lake is treated as a large chamber connected to channels or pipes between the compartments as shown in figure 1. Arrows represent the direction of flow through each channel or pipeline. When a pollutant enters the first lake, to know the rate of pollutant at which it enters the lake per unit time, $p(t)$ is introduced in the system of equations. The function $p(t)$ maybe a constant or it may vary with time.

Let the amount of pollution in each lake $i$ be denoted by $f_{i}(t)$ at any time $t \geq 0$, where $i=1,2,3$. It is being assumed that the amount of pollutant is equally distributed in each lake and the volume $V_{i}$ of each lake $i$ remain constant. Also the type of pollutant remains constant and not transforming into other kinds of pollutant. Then the concentration of the lake is given by

$$
C_{i}(t)=\frac{f_{i}(t)}{V_{i}}
$$

Initially each lake $f_{i}(t)$ is considered to be free of pollution i.e. $f_{i}(0)=0$ for $i=1,2,3$. So the following conditions are obtained:

$$
\begin{array}{ll}
\text { Lake } & 1: F_{13}=F_{21}+F_{31} \\
\text { Lake } & 2: F_{21}=F_{32} \\
\text { Lake } & 3: F_{31}+F_{32}=F_{13}
\end{array}
$$

Flux of pollutant flowing from lake $i$ to lake $j$ at any time $t$ measures the rate of flow of the concentration of pollutant and is given

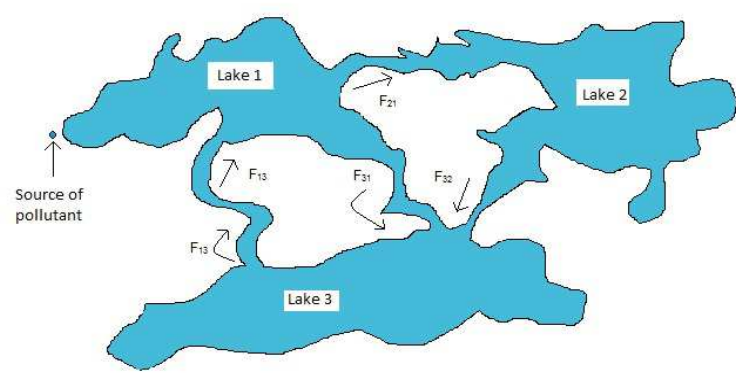

Fig. 1. System of three lakes connected with channels

by

$$
\text { Flux }_{j i}=f_{i} C_{i}(t)=\frac{(\text { flow rate })_{j i} f_{i}(t)}{V_{i}}
$$

Where $(\text { flow rate })_{j i}$ is a constant from lake $i$ to lake $j$. It can easily be seen that, Rate of change of pollutant= Input rate - output rate. From the above discussion, system of first-order differential equations is obtained as

$$
\begin{array}{r}
\frac{d x}{d t}=\frac{F_{13}}{V_{3}} z(t)+p(t)-\frac{F_{31}}{V_{1}} x(t)-\frac{F_{21}}{V_{1}} x(t) \\
\frac{d y}{d t}=\frac{F_{21}}{V_{1}} x(t)-\frac{F_{32}}{V_{2}} y(t) \\
\frac{d z}{d t}=\frac{F_{31}}{V_{1}} x(t)+\frac{F_{32}}{V_{2}} y(t)-\frac{F_{13}}{V_{3}} z(t)
\end{array}
$$

Now consider the models under discussion in specific cases.

\section{CASE 1: PERIODIC MODEL}

The Periodic model proves that the pollutant introduced in the lake is periodical. Example of this first case can be a factory producing waste and dumping it in the lakes. As working hours of factory is in the day time so mixing of pollutant stops during night. Let $p(t)=$ $1+\sin (t)$ in this case. Parameters are taken from Biazar [9]. $V_{1}=$ $2900 m i^{3}, V_{2}=850 m i^{3}, V_{3}=1180 m i^{3}, F_{21}=18 m i^{3} /$ year, $F_{32}=18 m i^{3} /$ year, $F_{31}=20 m i^{3} /$ year, $F_{13}=38 m i^{3} /$ year . So system Eq.(11a to 11c) becomes

$$
\begin{gathered}
\frac{d x}{d t}=\frac{38}{1180} z(t)+(1+\sin t)-\frac{38}{2900} x(t) \\
\frac{d y}{d t}=\frac{18}{2900} x(t)-\frac{18}{850} y(t) \\
\frac{d z}{d t}=\frac{20}{2900} x(t)+\frac{18}{850} y(t)-\frac{38}{1180} z(t)
\end{gathered}
$$

With the initial condition $x(0)=0, y(0)=0, z(0)=0$. As the system is solved using $P I A(1,1)$ therefore introducing perturbation parameter, Eq. (12a to 12c) becomes 


$$
\begin{gathered}
F_{1}=\dot{x}_{n+1}-\frac{38}{1180} z_{n+1} \epsilon-(1+\sin t) \epsilon+\frac{38}{2900} x_{n+1}=0 \\
F_{2}=\dot{y}_{n+1}-\frac{18}{2900} x_{n+1} \epsilon+\frac{18}{850} y_{n+1}=0 \\
F_{3}=\dot{z}_{n+1}-\frac{20}{2900} x_{n+1} \epsilon-\frac{18}{850} y_{n+1} \epsilon+\frac{38}{1180} z_{n+1}=0
\end{gathered}
$$

So for Eq. (7) this system becomes

$$
\begin{gathered}
\dot{x}_{n}+\frac{20}{2900} x_{n}+\frac{18}{2900} x_{n}+\dot{x}_{n}^{c} \epsilon+\frac{38}{2900} x_{n}^{c} \epsilon- \\
\frac{38}{1180} z_{n} \epsilon-\epsilon \sin t-\epsilon=0 \\
\dot{y}_{n}+\frac{18}{850} y_{n}+\dot{y}_{n}^{c} \epsilon+\frac{18}{850} y_{n}^{c} \epsilon-\frac{18}{2900} x_{n} \epsilon=0 \\
\dot{z}_{n}+\frac{38}{1180} z_{n}+\dot{z}_{n}^{c} \epsilon+\frac{38}{1180} z_{n}^{c} \epsilon-\frac{20}{2900} x_{n} \epsilon- \\
\frac{18}{850} y_{n} \epsilon=0
\end{gathered}
$$

For the convenience of calculations $\epsilon$ will be equal to one, but it's not necessary to take it equal to unity always. On taking $\epsilon=1$, then Eq.14a to 14c becomes

$$
\begin{gathered}
\dot{x}_{n}^{c}+\frac{38}{2900} x_{n}^{c}=-\dot{x}_{n}-\frac{20}{2900} x_{n}+\frac{18}{2900} x_{n}+ \\
\frac{38}{1180} z_{n}+\sin t+1 \\
\dot{y}_{n}^{c}+\frac{18}{850} y_{n}^{c}=-\dot{y}_{n}-\frac{18}{850} y_{n}+\frac{18}{2900} x_{n} \\
\dot{z}_{n}^{c}+\frac{38}{1180} z_{n}^{c}=-\dot{z}_{n}-\frac{38}{1180} z_{n}+\frac{20}{2900} x_{n}+\frac{18}{850} y_{n}
\end{gathered}
$$

Initial guess is same as the initial condition i.e. $x_{10}=0 ; y_{10}=0$; $x_{10}=0$. On solving with perturbation iteration method following iterations have been obtained

$$
\begin{gathered}
x_{11}=\frac{-1450 e^{\frac{-19 t}{1450}}}{3995459}\left[2075311-2102861 e^{\frac{19 t}{1450}}+\right. \\
\left.27550 e^{\frac{19 t}{1450}} \cos t-361 e^{\frac{19 t}{1450}} \sin t\right] \\
y_{11}=0 \\
z_{11}=0 \\
x_{12}=\frac{-1450 e^{\frac{-19 t}{1450}}}{3995459}\left[2075311-2102861 e^{\frac{19 t}{1450}}+\right. \\
\left.27550 e^{\frac{19 t}{1450}} \cos t-361 e^{\frac{19 t}{1450}} \sin t\right] \\
y_{12}=-\frac{425 e^{-\frac{9 t}{425}}}{1436778487093346}[-120141838562743+ \\
195761040073452 e^{\frac{199 t}{24650}}-75619920373334 e^{\frac{9 t}{425}}+ \\
\left.718862625 e^{\frac{9 t}{425}} \cos t+20964552291 e^{\frac{9 t}{425}} \sin t\right]
\end{gathered}
$$

$$
\begin{array}{r}
z_{12}=-\frac{2950 e^{-\frac{19 t}{590}}}{11374711823354683}[-41842326531549+ \\
104858917223795 e^{\frac{817 t}{42775}}-63017794035206 e^{\frac{19 t}{590}}+ \\
\left.1203342960 e^{\frac{19 t}{590}} \cos t+26548645394 e^{\frac{19 t}{590}} \sin t\right] \\
x_{13}=\frac{-1450 e^{\frac{-19 t}{150}}}{3995459}\left[2075311-2102861 e^{\frac{19 t}{1450}}+\right. \\
\left.27550 e^{\frac{19 t}{1450}} \cos t-361 e^{\frac{19 t}{1450}} \sin t\right]+ \\
\frac{725 e^{\frac{-19 t}{590}}}{1028535828821572438066709}[ \\
-25956636000675600198255- \\
31026272715604081619325 e^{\frac{817 t}{42775}}+ \\
569825943945469477380 e^{\frac{19 t}{590}}- \\
1242424450991475291127 t e^{\frac{817 t}{42775}}+ \\
314321732732340200 e^{\frac{19 t}{590}} \cos t- \\
\left.18376548600150620 e^{\frac{19 t}{590}} \sin t\right]
\end{array}
$$

$$
\begin{array}{r}
y_{13}=-\frac{425 e^{-\frac{9 t}{425}}}{1436778487093346}[-120141838562743+ \\
195761040073452 e^{\frac{199 t}{24650}}-75619920373334 e^{\frac{9 t}{425}}+ \\
\left.718862625 e^{\frac{9 t}{425}} \cos t+20964552291 e^{\frac{9 t}{425}} \sin t\right]
\end{array}
$$$$
z_{13}=-\frac{2950 e^{-\frac{19 t}{590}}}{11374711823354683}[-41842326531549+
$$$$
104858917223795 e^{\frac{817 t}{42775}}-63017794035206 e^{\frac{19 t}{590}}+
$$$$
\left.1203342960 e^{\frac{19 t}{590}} \cos t+26548645394 e^{\frac{19 t}{590}} \sin t\right]+
$$$$
\begin{aligned}
& \frac{2655 e^{-\frac{19 t}{590}}}{1431640896964162982626907}[ \\
& -10113672896070916202334+
\end{aligned}
$$$$
36801230771249690417465 e^{\frac{553 t}{50150}}
$$$$
34619157050905342366290 e^{\frac{817 t}{42775}}
$$$$
+7931528515036914031174 e^{\frac{19 t}{590}}+
$$$$
70660689654119985 e^{\frac{19 t}{590}} \cos t-
$$$$
\left.4703622674393601 e^{\frac{19 t}{590}} \sin t\right]
$$

$x_{14}, y_{14}, z_{14}$ are graphically shown in figures.

\section{CASE 2: LINEAR INPUT MODEL:}

Linear input describes the steady behavior of the pollutant addition into the lake. At time zero the pollutant concentration is also zero but as the time increases the addition of pollutant starts and remains steady afterwards. It can also be understood better by an example that if a manufacturing plant starts production and dump its raw wastage on a constant rate then $p(t)=100 t$. Eq. 111a to Eq. 11c 


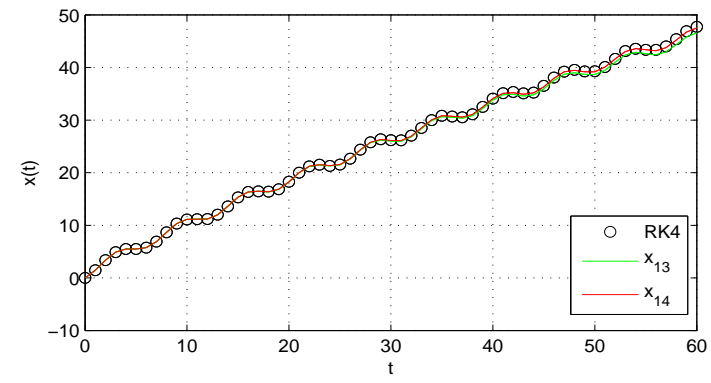

Fig. 2. Graphical representation of pollutant dumping periodically in Lake 1 with Perturbation iteration and $R K 4$ solutions

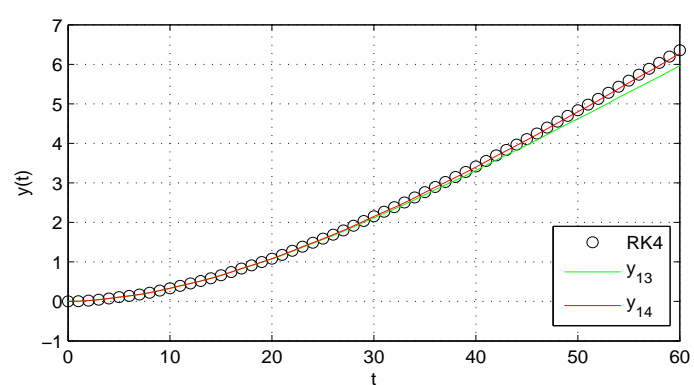

Fig. 3. Graph of mixing pollutant in Lake 2. Comparison of $R K 4$ and $P I A(1,1)$ approximation is shown.

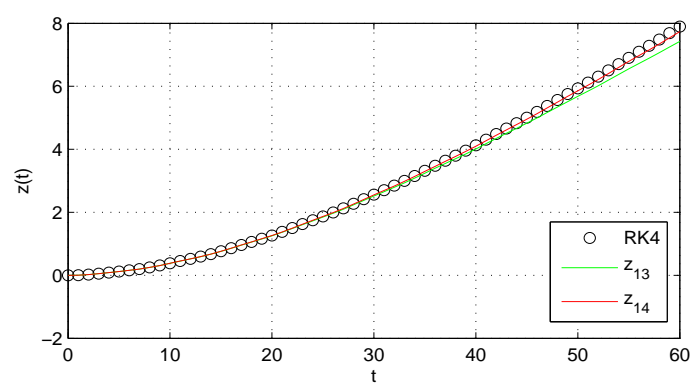

Fig. 4. Graphically pollutant mixing periodically in Lake 3 is presented by $R K 4$ and $P I A(1,1)$ approximations.

becomes

$$
\begin{gathered}
\frac{d x}{d t}=\frac{38}{1180} z(t)+100 t-\frac{38}{2900} x(t) \\
\frac{d y}{d t}=\frac{18}{2900} x(t)-\frac{18}{850} y(t) \\
\frac{d z}{d t}=\frac{20}{2900} x(t)+\frac{18}{850} y(t)-\frac{38}{1180} z(t)
\end{gathered}
$$

With the initial condition $x(0)=0, y(0)=0, z(0)=0$. As the system is solved using $P I A(1,1)$ therefore introducing perturbation parameter, Eq. [16a) to [16c) becomes

$$
\begin{gathered}
F_{1}=\dot{x}_{n+1}-\frac{38}{1180} z_{n+1} \epsilon-100 t \epsilon+\frac{38}{2900} x_{n+1}=0 \\
F_{2}=\dot{y}_{n+1}-\frac{18}{2900} x_{n+1} \epsilon+\frac{18}{850} y_{n+1}=0 \\
F_{3}=\dot{z}_{n+1}-\frac{20}{2900} x_{n+1} \epsilon-\frac{18}{850} y_{n+1} \epsilon+\frac{38}{1180} z_{n+1}=0
\end{gathered}
$$

So for Eq. 7 this system becomes

$$
\begin{gathered}
\dot{x}_{n}+\frac{20}{2900} x_{n}+\frac{18}{2900} x_{n}+\dot{x}_{n}^{c} \epsilon+ \\
\frac{38}{2900} x_{n}^{c} \epsilon-\frac{38}{1180} z_{n} \epsilon-100 t \epsilon=0 \\
\dot{y}_{n}+\frac{18}{850} y_{n}+\dot{y}_{n}^{c} \epsilon+\frac{18}{850} y_{n}^{c} \epsilon-\frac{18}{2900} x_{n} \epsilon=0 \\
\dot{z}_{n}+\frac{38}{1180} z_{n}+\dot{z}_{n}^{c} \epsilon+\frac{38}{1180} z_{n}^{c} \epsilon-\frac{20}{2900} x_{n} \epsilon- \\
\frac{18}{850} y_{n} \epsilon=0
\end{gathered}
$$

On taking $\epsilon=1$, then Eq. 18a to Eq. (18c) becomes

$$
\begin{gathered}
\dot{x}_{n}^{c}+\frac{38}{2900} x_{n}^{c}=-\dot{x}_{n}-\frac{20}{2900} x_{n}-\frac{18}{2900} x_{n}+ \\
\frac{38}{1180} z_{n}+100 t \\
\dot{y}_{n}^{c}+\frac{18}{850} y_{n}^{c}=-\dot{y}_{n}-\frac{18}{850} y_{n}+\frac{18}{2900} x_{n} \\
\dot{z}_{n}^{c}+\frac{38}{1180} z_{n}^{c}=-\dot{z}_{n}-\frac{38}{1180} z_{n}+\frac{20}{2900} x_{n}+\frac{18}{850} y_{n}
\end{gathered}
$$

Initial guess is same as the initial condition i.e. $x_{10}=0 ; y_{10}=0$; $x_{10}=0$. On solving with perturbation iteration method following iterations have been obtained

$$
\begin{aligned}
& x_{11}=\frac{145000 e^{-\frac{19 t}{1450}}}{361}\left[1450-1450 e^{\frac{19 t}{1450}}+19 e^{\frac{19 t}{1450}} t\right] \\
& y_{11}=0 \\
& z_{11}=0 \\
& x_{12}=\frac{145000 e^{-\frac{19 t}{1450}}}{361}\left[1450-1450 e^{\frac{19 t}{1450}}+19 e^{\frac{19 t}{1450}} t\right] \\
& y_{12}=\frac{42500 e^{-\frac{9 t}{425}}}{646551}\left[-2608225+6812100 e^{\frac{199 t}{24650}}-\right. \\
& \left.4203875 e^{\frac{9 t}{425}}+34029 e^{\frac{9 t}{425}} t\right] \\
& z_{12}=\frac{590000 e^{-\frac{19 t}{590}}}{294937}\left[-17405+105125 e^{\frac{817 t}{42775}}-\right. \\
& \left.87720 e^{\frac{19 t}{590}}+817 t e^{\frac{19 t}{590}}\right] \\
& x_{13}=\frac{145000 e^{\frac{-19 t}{1450}}}{361}\left[1450-1450 e^{\frac{19 t}{1450}}+19 e^{\frac{19 t}{1450}} t\right]+ \\
& \frac{725000 e^{\frac{-19 t}{590}}}{12682291}\left[1026895+11879125 e^{\frac{817 t}{42775}-}\right. \\
& 12906020 e^{\frac{19 t}{590}}+118465 t e^{\frac{817 t}{4275}}+70262 t e^{\frac{19 t}{590}}
\end{aligned}
$$




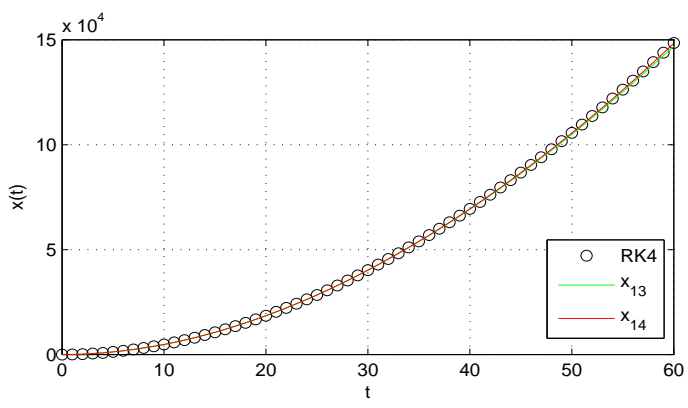

Fig. 5. At different time intervals the addition of linear input pollution with Perturbation iteration method and $R K 4$ method in Lake 1 is shown

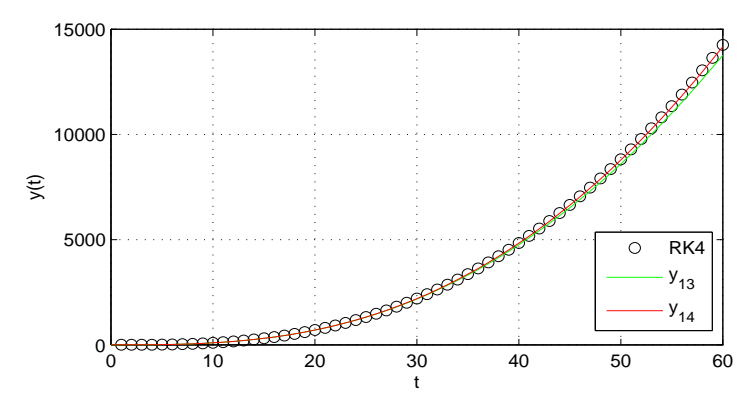

Fig. 6. Linear input of pollutant in Lake 2 is shown at different time intervals and compared by $R K 4$ and $P I A(1,1)$ approximations.

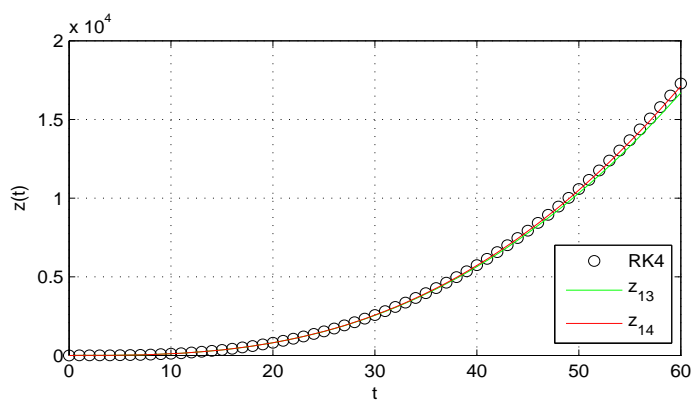

Fig. 7. Graphical representation of linear input pollutant in Lake 3 with Perturbation iteration method and $R K 4$ solutions

$$
\begin{array}{r}
y_{13}=\frac{42500 e^{-\frac{9 t}{425}}}{646551}\left[-2608225+6812100 e^{\frac{199 t}{24650}}-\right. \\
\left.4203875 e^{\frac{9 t}{425}}+34029 t e^{\frac{9 t}{425}}\right] \\
z_{13}=\frac{590000 e^{-\frac{19 t}{590}}}{294937}\left[\begin{array}{r}
-17405+105125 e^{\frac{817 t}{42775}}- \\
\left.87720 e^{\frac{19 t}{590}}+817 t e^{\frac{19 t}{590}}\right]
\end{array}\right.
\end{array}
$$

$x_{14}, y_{14}, z_{14}$ are graphically shown in figures.

\section{CASE 3: EXPONENTIALLY DECAYING MODEL}

This model is assumed when heavy dumping of pollutant is under consideration i.e. $p(t)=200 e^{-10 t}$. Example of such a case is if any industry situated in a city collects and stores its wastage and dumps it after a few days' period. So Eq.(11a) to Eq.(11c) takes the form

$$
\begin{gathered}
\frac{d x}{d t}=\frac{38}{1180} z(t)+200 e^{-10 t}-\frac{38}{2900} x(t) \\
\frac{d y}{d t}=\frac{18}{2900} x(t)-\frac{18}{850} y(t) \\
\frac{d z}{d t}=\frac{20}{2900} x(t)+\frac{18}{850} y(t)-\frac{38}{1180} z(t)
\end{gathered}
$$

With the initial condition $x(0)=0, y(0)=0, z(0)=0$. As the system is solved using $P I A(1,1)$ therefore introducing perturbation parameter, Eq. 20a to 20c becomes

$$
\begin{gathered}
F_{1}=\dot{x}_{n+1}-\frac{38}{1180} z_{n+1} \epsilon+200 e^{-10 t} \epsilon+\frac{38}{2900} x_{n+1}=0 \\
F_{2}=\dot{y}_{n+1}-\frac{18}{2900} x_{n+1} \epsilon+\frac{18}{850} y_{n+1}=0 \\
F_{3}=\dot{z}_{n+1}-\frac{20}{2900} x_{n+1} \epsilon-\frac{18}{850} y_{n+1} \epsilon+\frac{38}{1180} z_{n+1}=0
\end{gathered}
$$

So for Eq. (7) this system becomes

$$
\begin{gathered}
\dot{x}_{n}+\frac{20}{2900} x_{n}+\frac{18}{2900} x_{n}+\dot{x}_{n}^{c} \epsilon+\frac{38}{2900} x_{n}^{c} \epsilon- \\
\frac{38}{1180} z_{n} \epsilon+200 \epsilon e^{-10 t}=0 \\
\dot{y}_{n}+\frac{18}{850} y_{n}+\dot{y}_{n}^{c} \epsilon+\frac{18}{850} y_{n}^{c} \epsilon-\frac{18}{2900} x_{n} \epsilon=0 \\
\dot{z}_{n}+\frac{38}{1180} z_{n}+\dot{z}_{n}^{c} \epsilon+\frac{38}{1180} z_{n}^{c} \epsilon-\frac{20}{2900} x_{n} \epsilon- \\
\frac{18}{850} y_{n} \epsilon=0
\end{gathered}
$$

On taking $\epsilon=1$, then Eq. 22a to Eq. (22c) becomes

$$
\begin{gathered}
\dot{x}_{n}^{c}+\frac{38}{2900} x_{n}^{c}=-\dot{x}_{n}-\frac{20}{2900} x_{n}-\frac{18}{2900} x_{n}+ \\
\frac{38}{1180} z_{n}-200 e^{-10 t} \\
\dot{y}_{n}^{c}+\frac{18}{850} y_{n}^{c}=-\dot{y}_{n}-\frac{18}{850} y_{n}+\frac{18}{2900} x_{n} \\
\dot{z}_{n}^{c}+\frac{38}{1180} z_{n}^{c}=-\dot{z}_{n}-\frac{38}{1180} z_{n}+\frac{20}{2900} x_{n}+\frac{18}{850} y_{n}
\end{gathered}
$$

Initial guess is same as the initial condition i.e. $x_{10}=0 ; y_{10}=0$; $x_{10}=0$. On solving with perturbation iteration method following iterations have been obtained

$$
\begin{gathered}
x_{11}=\frac{290000 e^{-10 t}}{14481}\left[-1+e^{\left.\frac{14481 t}{1450}\right]}\right. \\
y_{11}=0 \\
z_{11}=0 \\
x_{12}=\frac{290000 e^{-10 t}}{14481}\left[-1+e^{\frac{14481 t}{1450}}\right]
\end{gathered}
$$




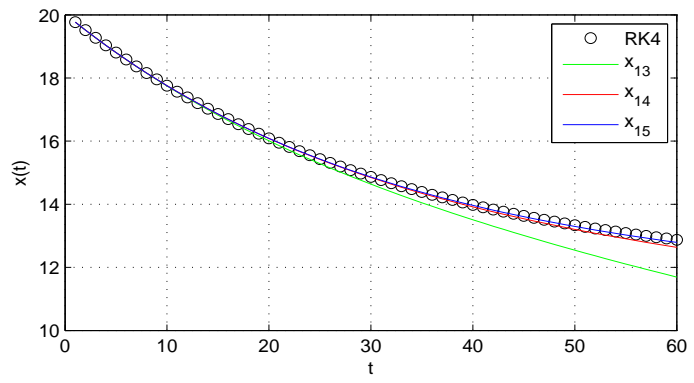

Fig. 8. Heavy dumping of pollutant in Lake 1 .Solutions obtained by Perturbation iteration method and $R K 4$ method

$$
\begin{gathered}
y_{12}=\frac{85000 e^{-10 t}}{1357930031}\left[199-246177 e^{\frac{4241 t}{425}}+\right. \\
\left.245978 e^{\frac{14481 t}{1450}}\right] \\
z_{12}=\frac{590000 e^{-10 t}}{69577975737}\left[1634-854379 e^{\frac{5881 t}{590}}+\right. \\
\left.852745 e^{\frac{14481 t}{1450}}\right] \\
x_{13}=\frac{290000 e^{-10 t}}{14481}\left[-1+e^{\frac{14481 t}{1450}}\right]+ \\
\frac{145000 e^{-10 t}}{43325022665842371}[-13349780+ \\
3649817378205 e^{\frac{5881 t}{590}}- \\
3649804028425 e^{\frac{14481 t}{1450}}+ \\
\left.69577975737 t e^{\frac{14481 t}{1450}}\right] \\
y_{13}=\frac{85000 e^{-10 t}}{1357930031}\left[199-246177 e^{\frac{4241 t}{425}}+\right. \\
\frac{12300 e^{-\frac{19 t}{590}}}{1357930031}\left[-\frac{117410 e^{-\frac{5881 t}{590}}}{5881}-\right. \\
\left.245978 e^{\frac{14481 t}{1450}}\right] \\
z_{13}=\frac{590000 e^{-10 t}}{69577975737}\left[1634-854379 e^{\frac{5881 t}{590}}+\right. \\
\left.852745 e^{\frac{14481 t}{1450}}\right]+\frac{33271398000 e^{-\frac{19 t}{590}}}{2657041681}+
\end{gathered}
$$

$x_{14}, y_{14}, z_{14}$ and $x_{15}, y_{15}, z_{15}$ are graphically shown in figures.

\section{CONCLUSION}

In this paper, a perturbation iteration method has been used to analyze the pollution rate in system of lakes. Results are compared by $R K 4$ method as shown in figures. Three different types of pollutant have been assumed and the convergence of this system is checked that yielded satisfying results. This system of equations has already been analyzed by different methods such as HPM and VIM but this method is more accurate and easy to solve such type of systems

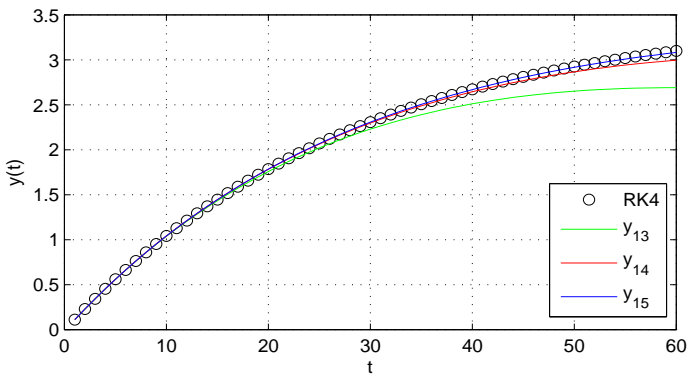

Fig. 9. Graphically shown heavy dumping of pollutant in Lake 2 with Perturbation iteration method and $R K 4$ solutions

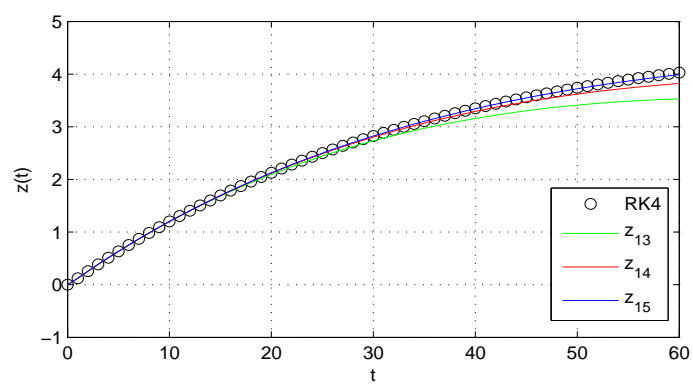

Fig. 10. Graphical comparison of heavy dumping of pollutant in Lake 3 by $R K 4$ and $P I A(1,1)$.

because more than one corrected terms can accelerate the process of convergence. However we have only considered $P I A(1,1)$ and get the required convergence on second iteration in some models. PIA method obtains its coefficients using an easily computable straightforward procedure that can be implemented into programs like Matlab or Mathematica.

\section{CONFLICT OF INTERESTS}

The authors declare that there is no conflict of interests regarding the publication of this paper.

\section{ACKNOWLEDGMENT}

We thank the reviewers for their thorough efforts in editing our paper and highly appreciate the comments and constructive criticism that significantly contributed in improving the quality of the publication. The authors also thank Ms.Wishaal Khalid for proofreading our research paper.

\section{REFERENCES}

[1] Pakdemirli, M. and Boyaci,H. (2007) Generation of root finding algorithms via perturbation theory and some formulas, Applied Mathematics and Computation, 184. pp 783-788

[2] Pakdemirli, M., Boyaci, H. and Yurtsever, M.A. (2007) Perturbative derivation and comparisons of root-finding algorithms with fourth order derivatives, Mathematical and Computational Applications, 12. pp 117-124

[3] Pakdemirli, M., Boyaci, H. and Yurtsever, H.A. (2008) A root finding algorithm with fifth order derivatives, Mathematical and Computational Applications, 13. pp 123-128 
[4] Pakdemirli, M., Aksoy, Y. and Boyaci, H. (2011) A New Perturbation-Iteration Approach for First Order Differential Equations, Mathematical and Computational Applications, 16. pp 890-899

[5] Aksoy, Y. and Pakdemirli, M. (2010) New PerturbationIteration Solutions for Bratu-type Equations, Computers \& Mathematics with Application, 59. pp 2802-2808

[6] Aksoy, Y., Pakdemirli, M., Abbasbandy, S. and Boyaci, H. (2012) New Perturbation-Iteration Solutions for Nonlinear Heat Transfer Equations, International Journal of Numerical Methods for Heat \& Fluid Flow, 22. pp 814-828

[7] Dolapci, I.T., enol, M. and Pakdemirli, M. (2013) New perturbation iteration solutions for Fredholm and Volterra integral equations, Journal of Applied Mathematics, Article ID: 682537, 5 pages, http://dx.doi.org/10.1155/2013/682537.

[8] Senol, M., Dolapci, I.T., Aksoy, Y. and Pakdemirli, M. (2013) Perturbation-Iteration method for first order differential equations and systems, Abstract and Applied Analysis, Article ID: 704137, 6 pages, http://dx.doi.org/10.1155/2013/704137

[9] Biazar, J., Farrokhi, L. and Islam, M.R. (2006) Modeling the pollution of a system of lakes, Applied Mathematics and Computation, 178. pp 423-430 